\title{
Monographies des plantes à haute fréquence d'utilisation en pharmacopée traditionnelle au Moyen Atlas Central Maroc.
}

\author{
Amine DAOUDI ${ }^{1}$, Mariame NAJEM ${ }^{1}$, Lamia BACHIRI ${ }^{1}$, Jamal IBIJBIJEN ${ }^{1}$ et Laila \\ NASSIRI $^{1}$ \\ ${ }^{1} E$ quipe de Microbiologie du Sol et de l'Environnement, Faculté des Sciences- Université Moulay Ismail -Meknès \\ Auteur correspondant; E-mail : Lamiaebachiri@gmail.com, Faculté des Sciences; B.P : 11201, Meknès, Maroc. Tel: (+212) \\ 618859436
}

Mots-clés : Monographies- Plantes médicinales - Moyen Atlas- Maroc.

Keywords: Monographs-Medicinal plants-Middle Atlas-Morocco.

Publication date 30/06/2019, http://www.m.elewa.org/JAPS;

\section{RÉSUMÉ}

La région du Moyen Atlas central recèle d'un grand potentiel floristique, qui a permis au fil du temps, l'acquisition d'un important savoir quant à l'utilisation des plantes en pharmacopée traditionnelle. Aussi, le présent travail consiste en l'élaboration de monographies de 11 plantes parmi les plus utilisées en phytothérapie locale; celles-ci sont le fruit d'enquêtes ethnobotaniques menées auprès de nombreux acteurs de la médecine traditionnelle au Moyen Atlas central et d'investigations phytochimiques et biologiques entamées au laboratoire.

\begin{abstract}
In Morocco, the central Middle Atlas region has a great floristic potential, which over time has led to the acquisition by population of an important knowledge about the use of plants in traditional pharmacopoeia. So, the present work consists of the elaboration of monographs of 11 plants among the most used in local herbal medicine; these are the result of ethnobotanical surveys carried out with numerous actors of traditional medicine in the central middle Atlas and of phytochemical and biological investigations started in the laboratory.
\end{abstract}

\section{INTRODUCTION}

Au Maroc, le Moyen Atlas central, ensemble de plateaux karstiques constitue une écorégion étalée sur une superficie d'environ 1200000 ha et un gradient altitudinal allant de $2000 \mathrm{~m}$ au Nord à $800 \mathrm{~m}$ au Sud; cette situation géographique confère à la région une grande variété bioclimatique, exprimée par un important couvert forestier et une flore remarquable (Benbrahim et al., 2004). Ce potentiel forestier est un enjeu stratégique pour la région, du fait qu'il constitue un patrimoine protecteur et productif, un réservoir génétique de biodiversité et est donc un espace socioéconomique important en plus d'être récréatif et culturel (Boujrouf, 2004). Aussi, l'originalité de la flore régionale réside sa richesse en plantes aromatiques et médicinales dont plusieurs sont endémiques, et peuvent être valorisées en tant que sources de produits à forte valeur ajoutée (Hmamouchi, 1999). De son côté, la population du Moyen Atlas central à dominance rurale, vivant dans des milieux à 
vocation surtout pastorale et sylvopastorale reste attachée à la nature, au contact des plantes ; là, l'observation liée à l'expérience et la transmission des informations glanées au cours $\mathrm{du}$ temps font que certaines personnes deviennent capables de connaitre les plantes, de retrouver celles qui soignent, et repérer celles indésirables, voire toxiques (Bellakhdar, 1997). Pour toutes ces raisons, et à l'aide de fiches questionnaires (Annexe 1), nous avons entrepris des investigations ethnobotaniques durant la période ((janvier-juillet 2013) auprès d'un ensemble d'acteurs de la médecine traditionnelle, englobant, herboristes, droguistes et tradipraticiens; le but étant de recueillir le maximum d'informations sur les plantes utilisées en phytothérapie régionale. Chaque répondant a délivré une moyenne de 18 plantes, et 1042 fiches questionnaires furent ainsi remplies. Au total, 125 plantes médicinales ont été répertoriées, et sont réparties en 111 genres et 52 familles. Par la suite, onze «11» plantes ont été retenues sur la base de leur fréquence de citations, pour des analyses chimiques aussi bien qualitatives que quantitatives et une évaluation du pouvoir antimicrobien de leurs extraits aqueux et de leurs huiles essentielles. Auparavant, des prospections sur le terrain ont été réalisées pour la localisation des plantes choisies et la récolte d'échantillons pour l'expérimentation au laboratoire. Dans ce qui suit, nous présentons les résultats obtenus sous forme de monographies, où la systématique, la description et la répartition de chaque plante sont précisées en s'appuyant essentiellement sur la flore pratique du Maroc de Fennane et al. (1999, 2007 et 2014). Pour leur part, toutes les informations concernant l'usage des plantes, notamment, les noms vernaculaires, la partie utilisée, les doses utilisées, la durée d'utilisation, les indications thérapeutiques, la toxicité, émanent de l'enquête ethnobotanique conduite dans la région. Quant aux données relatives à la composition chimique et certaines activités biologiques, elles émanent des travaux menés au laboratoire de Microbiologie du Sol et de l'Environnement, à la Faculté des Sciences de Meknès.

\begin{tabular}{|c|c|}
\hline $\begin{array}{l}\text { Systématique } \\
\text { - Embranchement : Spermaphytes } \\
\text { - Sous-embranchement : Angiospermes } \\
\text { - Classe : Dicotylédones } \\
\text { - Ordre : Campanales } \\
\text { - Famille : Asteracées } \\
\text { - Genre : Calendula L. } \\
\text { - Espèce : Calendula arvensis L. } \\
\text { - Nom français : Soucis } \\
\text { - Nom arabe : جمرة:Tamra } \\
\text { - Nom berbère -Tajmirt }\end{array}$ & $\begin{array}{l}\text { Ethnobotanique : Utilisée surtout en } \\
\text { thérapeutique; les feuilles, les fleurs et les racines } \\
\text { sont les parties les plus exploitées, et sont utilisées } \\
\text { sous forme de décoction ou macération pendant } \\
\text { une semaine sans risque de toxicité ; cette plante } \\
\text { est recommandée pour traiter les problèmes } \\
\text { rhumatismaux et dermiques (Daoudi et al.,2015,a). } \\
\text { Autrefois, elle était utilisée en cuisine, en médecine } \\
\text { et en cosmétique par les civilisations indiennes, } \\
\text { arabes et grecques de l'antiquité (Bellakhdar, } \\
\text { 1997). Les fleurs et les racines de cette plante sont } \\
\text { employées pour traiter les problèmes } \\
\text { d'inflammations de la peau, eczéma et des } \\
\text { muqueuses de la bouche et de la gorge, les plaies et } \\
\text { les ulcères variqueux, et, elle est antiseptique, } \\
\text { antifongique, antivirale, cholérétique et } \\
\text { emménagogue. Aussi, plusieurs travaux } \\
\text { scientifiques ont été effectués pour confirmer } \\
\text { certains usages traditionnels de cette plante } \\
\text { comme les propriétés anti-inflammatoires, } \\
\text { antiseptiques, antibactériennes et emménagogues } \\
\text { (Hmamouchi, 1999). Des travaux récents } \\
\text { rapportent que Calendula arvensis L. stimule la }\end{array}$ \\
\hline
\end{tabular}




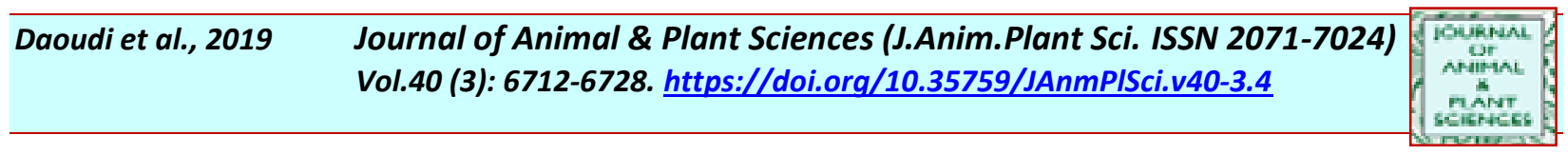

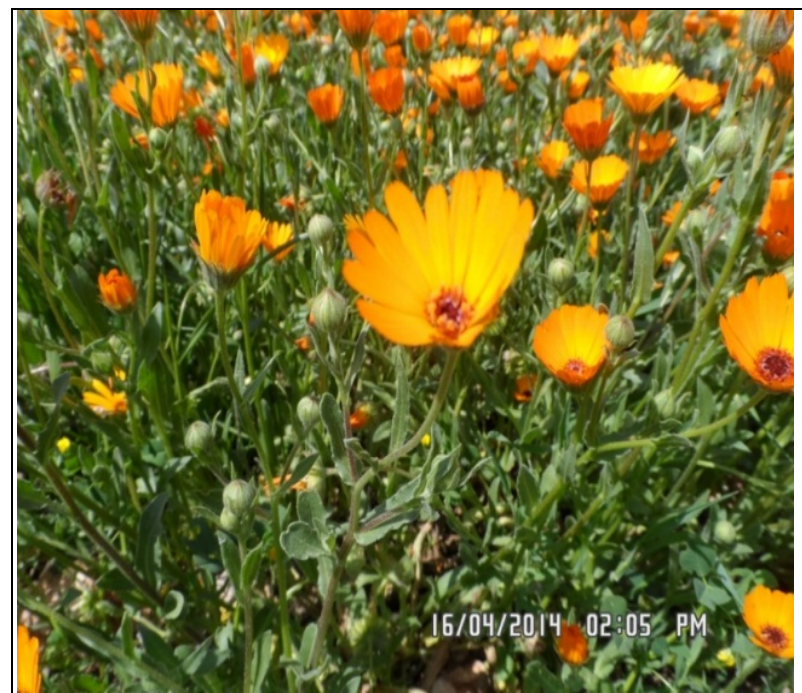

Description botanique : Plante herbacée annuelle de 30 à 50 centimètres de haut, à tige anguleuse, velue et rameuse, portant des feuilles alternes entières ou légèrement dentées. Les capitules terminaux, solitaires sont larges (3 à 7 centimètre), avec un involucre de 2 rangs de bractées velues. Les fruits sont des akènes très courbés et hérissés d'épines sur leur face dorsale. Période de floraison: été. Période de récolte: août (Hmamouchi, 1999 ; Fennane et al., 2014).

- Ecologie/Répartition: Le souci est cultivé partout au Maghreb comme plante ornementale. On trouve aussi à l'état spontané, des espèces sauvages $(C$. algeriensis Boiss., C. arvensis L., C. murbeckii Lanza) dont les capitules, plus petits, peuvent être employés, en usage externe, de la même façon que l'espèce cultivée (Fennane et al., 2014). pression du ventricule gauche, le flux aortique, et réduit la taille d'un éventuel infarctus myocardique, et par la suite cause l'apoptose des cardiomyocytes; elle modifie le signal létal par ischémie en un signal de survie en modifiant la voie de l'oxydation et de l'inflammation (Ghédira et Goetz, 2016).

- $\quad$ Phytochimie : Cette plante contient une teneur moyenne en tanins et en flavonoïdes, en plus des caroténoïdes, Stérols et triterpènes. D'après Ghédira et Goetz (2016), elle contient des flavonoïdes, des saponines tri terpéniques, des Alcools tri terpéniques, des caroténoïdes et des xanthophylles.

Comparé au décocté et macéré, l’infusé de Calendula arvensis $\mathrm{L}$. renferme la quantité la plus importante de polyphénols (68,69 Qteq Ag mg / $1 \mathrm{~g}$ d'extrait). (Daoudi et al. ,2017- a)

- $\quad$ Activité biologique : Les extraits aqueux de cette plante, testés sur des souches de champignons phytopathogènes, notamment Fusarium oxysporum, Penicillium expansum n'ont aucun effet anti-mycotique (Daoudi et al. ,2017-a). D'après Bellakhdar (2006), cette plante possède surtout des propriétés antibactériennes, cicatrisantes et anti-inflammatoires. 


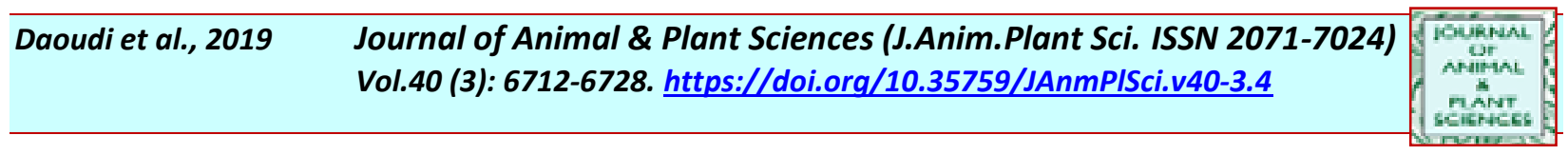

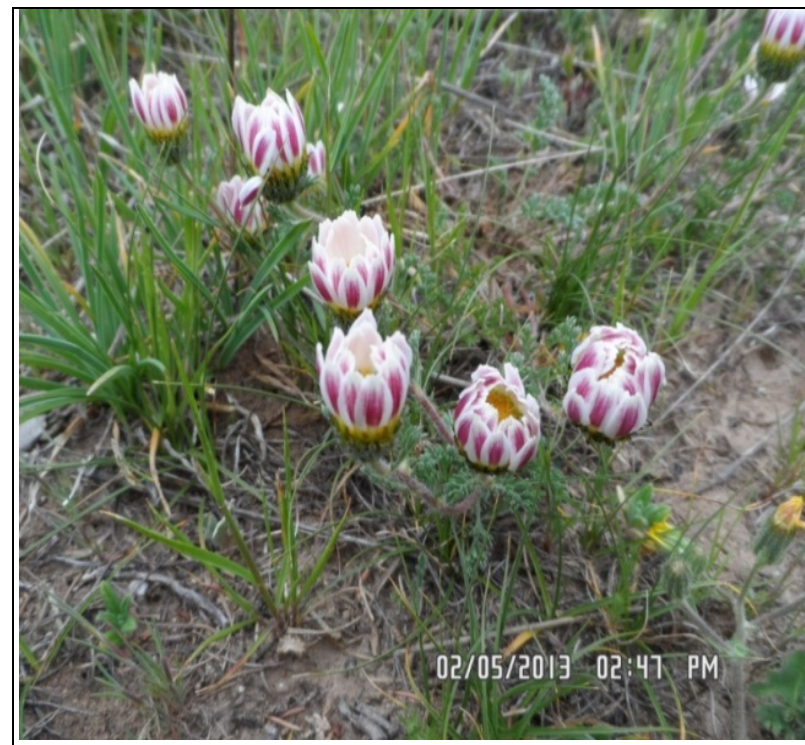

\section{Systématique}

- Embranchement : Spermaphytes

- Sous-embranchement : Angiospermes

- Classe : Dicotylédones

- Ordre : Campanales

- Famille : Asteracées

- Genre: Anacyclus L.

-Espèce : Anacyclus pyrethrum (L) Link

- Nom français : Pyrèthre d'Afrique

- Nom arabe : عقبر قرحة : Akirkarha

- Nom berbère : تكندزت : Tiguandizt

- Description botanique: Plante vivace; Tiges couchées en cercle sur le sol, à rameaux monocéphales ou polycéphales, et à racine pivotante, développée, brune et d'odeur aromatique. Fleurs à ligules blanches et plus ou moins pourprées à l'extérieur. Capitules hétérogames. Akènes tous chauves ou à couronne peu marquée. (Hmamouchi, 1999 ; Fennane et al, 2014).

- Ecologie/Répartition : Espèce endémique du Maroc et de l'Algérie. Se trouve dans les clairières des forêts, matorrals, ermes, pâturages ; plaines basses, moyennes et hautes montagnes, en semi-aride froid, subhumide, humide, haute montagnes, au niveau de l'Atlas saharien, Anti Atlas, Haut Atlas, Moyen Atlas, Maroc atlantique nord, plateaux du Maroc oriental (Jerada) Rif (Chaouène; jbelAssilenh, vallée de Tizi-n-lel) (Fennane et al., 2014).
Ethnobotanique: La racine est la partie la plus exploitée ; elle est prise sous forme de décoction à base d'une pincée. Cette plante est largement utilisée pour traiter les problèmes rhumatismaux, gastriques, respiratoires et bucco-dentaires (Daoudi et al., 2015, a). La poudre de racine est conseillée en usage interne contre les maladies du foie, la décoction est utilisée pour soigner des cas de bronchites et l'énurésie (Sijelmassi, 1996; Daoudi et al., 2014). Conseillé pour traiter des pathologies bucco-dentaires (Bellakhdar, 1997), A. pyrethrum est utilisé dans la fabrication de dentifrices (Sijelmassi, 1996).

- Phytochimie : L'analyse phytochimique réalisé sur l'extrait de racines broyées d'Anacyclus pyrethrum a révélé la présence d'alcaloïdes, de flavonoïdes, de tanins, de saponines; ces résultats concordent avec ceux d'El Azzouzi et al. (2014). La bibliographie signale la présence dans les racines d'A.pyrethrum de composés polyacétyléniques, de lignanes d'alkamides, notamment la pellitorine, dont la présence est associée à l'effet sialagogue de la plante, connu depuis longtemps (Hamimed, 2009).

D’après le dosage des polyphénols des extraits aqueux issus respectivement de la macération, la décoction et l'infusion, le macéré présente la quantité équivalente d'acide gallique en $\mathrm{mg}$ par $1 \mathrm{~g}$ d'extrait soit (97,57 Qteq Ag mg / $1 \mathrm{~g}$ d'extrait), la plus importante par rapport aux autres extraits (Daoudi et al., 2017- b).

- Activité biologique: Staphylococus aureus, Escherichia coli et Klebseilla pneumoniae sont sensibles au macérât d'Anacyclus pyretbrum utilisé à $100 \mu \mathrm{g} / \mathrm{ml}$ (Daoudi et al., 2017, b). Ces résultats concordent avec ceux de (El Azzouzi et al., 2014). L'extrait aqueux d'Anacyclus pyrethrum produit des effets antibactériens et antifongiques, respectivement sur, Listeria monocytogenes et Candida albicans (Selles et al., 2012). 


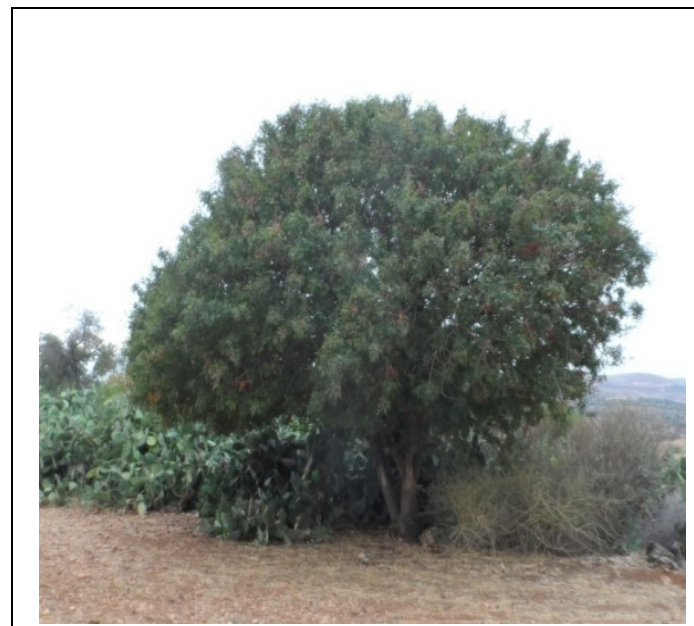

\section{Systématique}

- Embranchement : Spermaphytes

- Sous-embranchement : Angiospermes

- Classe : Dicotylédones

- Ordre : Sapindales

- Famille : Anacardiacées

- Genre : Pistacia

- Espèce : Pistacia atlantica Desf

- Nom français : Pistachier de l'Atlas

- Nom arabe :Lebtem

- Nom berbère : تدس -Tadis

- Description botanique: Espèce souvent dioïque, ligneuse, pouvant atteindre 10 à 20 mètre de haut, à tronc bien individualisé, à frondaison hémisphérique et ramifications étalées. Le rameau jeune est rougeâtre à feuillage caduque, composé, imparipenné à 7 ou 9 folioles ovales acuminées. Les fleurs réunies en grappes lâches sont apétales et rougeâtres; le fruit est une drupe (Hmamouchi, 1999 ; Fennane et al.,, 2007).

- Ecologie/Répartition: Réparti dans tout le Maroc, le pistachier de l'Atlas se localise dans les forêts des basses et moyennes montagnes jusque vers $2000 \mathrm{~m}$, et tolère des bioclimats : aride, semi-aride doux, semi-aride froid, subhumide (Hmamouchi, 1999; Fennane et al., 2007).
- Ethnobotanique: Pistacia atlantica, plante médicinale par excellence est aussi utilisée dans le domaine cosmétique par certaines personnes. En phytothérapie, les parties de la plante les plus utilisées, sous forme de décoction, sont les graines, la résine et les feuilles. Une cuillère de la poudre des graines ou des feuilles séchées pendant une semaine est la formule la plus recommandée dans la région d'étude ; mais, la prudence s'impose, vu le risque d'intoxication (Daoudi et al., 2015-a).

Cette plante est utilisée pour traiter les affections bucco-dentaires, les maladies de la peau, les affections du tube digestif, les maladies respiratoires, les pathologies neurologiques (Hmamouchi, 1999 ; Daoudi et al., 2013 ; Daoudi et al., 2015-b). L'usage traditionnel de la résine, comme masticatoire (chewing- gum) contre les infections stomacales et comme antiseptique pour les voies respiratoires est très courant (Giner-Larza et al., 2001). La plante est également utilisée dans les préparations de certains produits cosmétiques, tel le célèbre savon de Taza au Maroc (Daoudi et al., 2013).

- Phytochimie: l'étude phytochimique révèle la présence de quelques groupes chimiques biologiquement actifs : les tanins, les flavonoïdes, les stérols et les triterpènes; ces résultats concordent avec ceux de (Maamri, 2008). Le pistachier de l'Atlas se caractérise par la présence d'une forte teneur en composés monoterpéniques, des flavonoïdes, des flavones, des gallotanins, et des phénols simples tels que l'acide gallique et l'acide $\mathrm{p}$-coumarique (Benabderrahmane et al., 2009). D'autres groupes chimiques caractérisent l'huile essentielle des fruits du pistachier de l'Atlas, à savoir, les triterpénoides, les acides gras insaturés tels les acides oléique, linoléique, palmitique, palmitoléique, stéarique, linolénique, les stéroïdes et les triglycérides (Benhassaini et al., 2007)

- $\quad$ Activité biologique : L'huile essentielle extraite des feuilles de $P$. atlantica est dotée d'une forte activité anti-microbienne vis-à-vis de Stapbylococus aureus. Ces résultats concordent avec ceux de (Ghalem et Benali, 2009). 


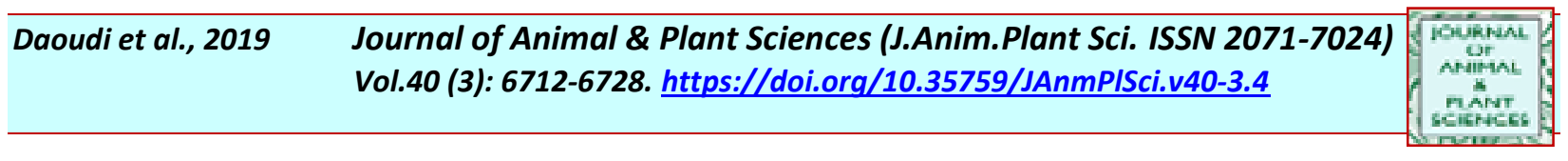

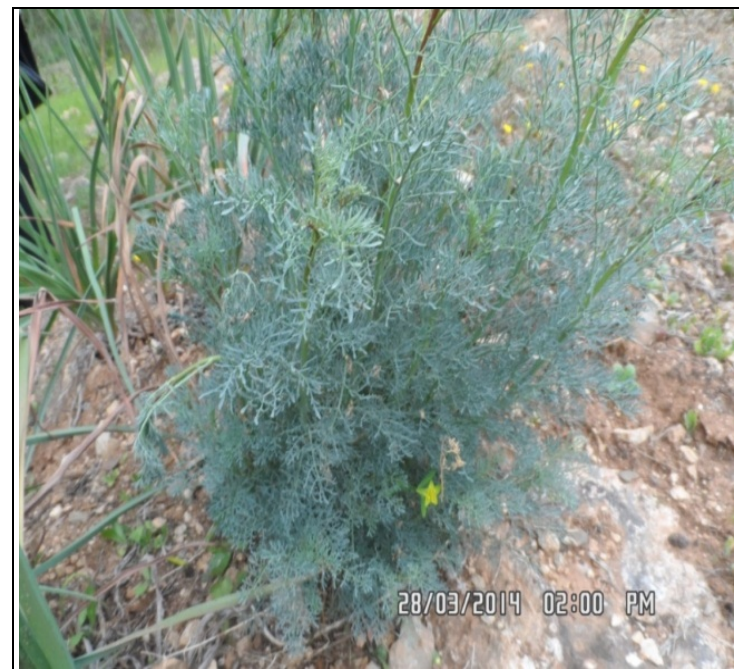

\section{Systématique}

- Embranchement : Spermaphytes

- Sous-embranchement: Angiospermes

- Classe : Dicotylédones

- Ordre : Rutales

- Famille : Rutacées

- Genre : Ruta

- Espèce : Ruta montana (L.) L

- Nom français : Rue des montagnes

- Nom arabe : الفيجل-Figel

- Nom berbère : أورني-Awerni

- Description botanique : Plante herbacée à feuilles triangulaires divisées en lobes ovales; les fleurs sont jaunes, le fruit est une capsule à graines réniformes. (Hmamouchi, 1999).

- Ecologie/Répartition : la rue des montagnes se rencontre dans les forêts claires, les matorrals rocailleux et les basses et moyennes montagnes, en bioclimats: aride, semi-aride doux, semi-aride froid, subhumide.

Sa répartition est large, entre l'Anti Atlas, Haut Atlas, Moyen Atlas, Maroc atlantique moyen, Maroc atlantique nord, plateaux du Maroc oriental, littoral de la méditerranée et le Rif (Hmamouchi, 1999 ; Fennane et al., 2007).
- Ethnobotanique: Ruta montana est une plante à usage thérapeutique par excellence; les parties les plus utilisées sont les feuilles et les racines. Cette plante est utilisée sous différentes préparations à savoir la décoction, l'infusion et la macération ; de plus, ses huiles essentielles ont aussi des vertus médicinales. Une pincée pendant une semaine est la posologie la plus recommandée, pour éviter d'éventuelles intoxications (Daoudi et al., 2015-a). Elle est sollicitée pour traiter plusieurs pathologies à savoir: les affections dermatologiques, les affections respiratoires, bucco-dentaires, urogénitales, neurologiques, osteo-articulaires et les affections du tube digestif, (Bellakhdar et al., 1991 ; Hmamouchi, 1999; Lahsissene et al., 2009 ; Daoudi et al., 2015-a; Daoudi et al., 2015-b; Daoudi et al., 2016). Cette plante est le remède des mauvais effets du traumatisme sur les muscles et les tendons (Mouhib et El Omari, 1997).

- Phytochimie : Les tests chimiques réalisés sur cette espèce révèlent la présence de dérivés phénoliques, surtout des tanins galliques et catéchiques, des leucoanthocyanes et des flavanones (Daoudi et al., 2016). a révélé La présence d'alcaloïdes (révélée par Le test avec le réactif de Mayer) explique la toxicité de R. montana.

- $\quad$ Activité biologique : L'huile essentielle de R. montana possède un effet important sur Staphylococus aureus alors qu'aucun effet n'est observé sur Escherichia coli et Klebseilla pneumoniae (Daoudi et al., 2016). Ces résultats concordent avec ceux de (Belkassam et al., 2011). 

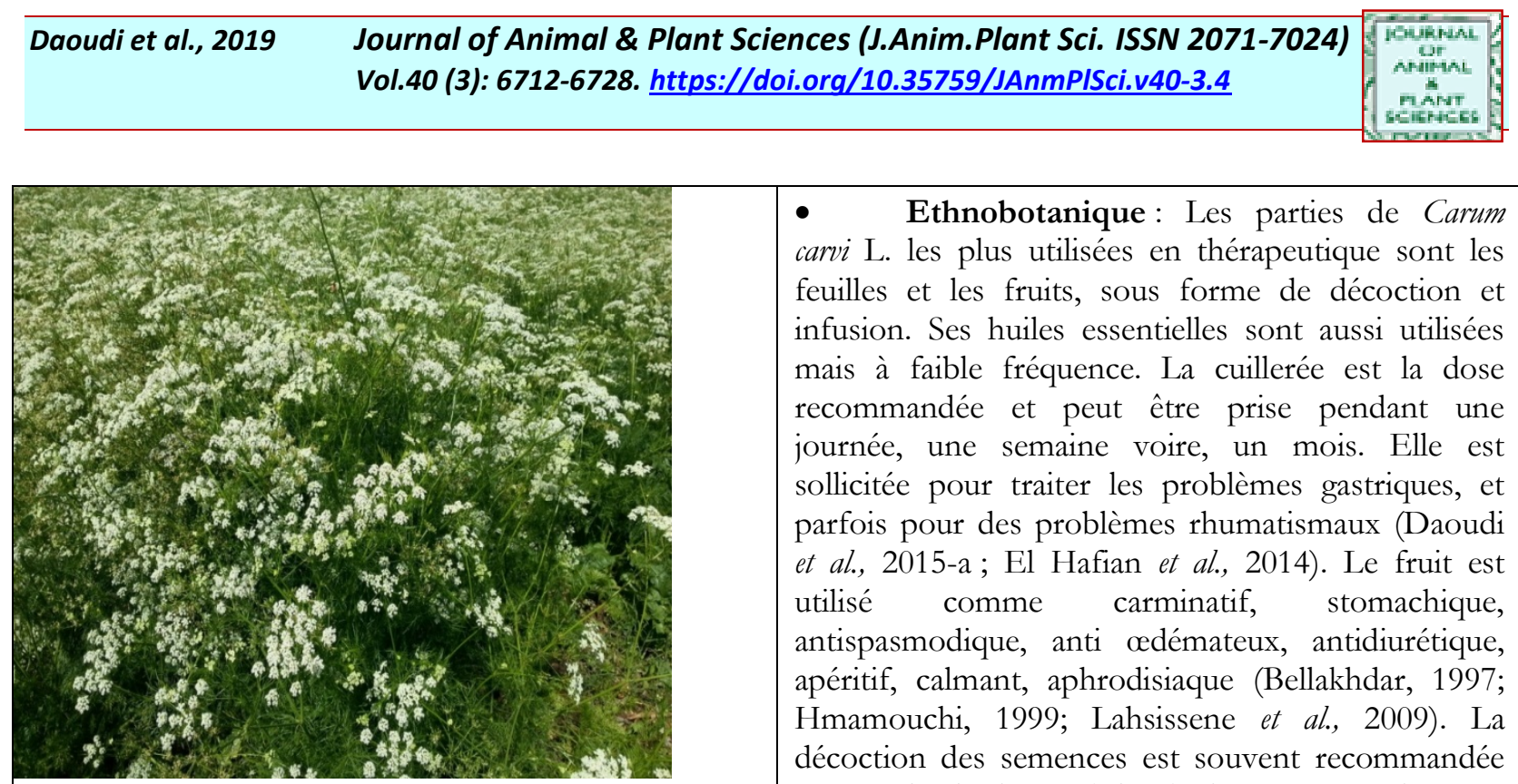

\section{Systématique}

- Embranchement : Spermaphytes

- Sous-embranchement : Angiospermes

- Classe : Dicotylédones

- Ordre : Apiales

- Famille : Apiacées

- Genre : Carum L.

- Espèce : Carum carvi L.

- Nom français : Carvi

- Nom arabe : كرويةkerwiya

- Nom berbère : تقروبة -Tikiro

\section{$22 / 05 / 2014$}

- Description botanique : Plante bisannuelle dégageant une odeur aromatique et possédant une racine pivotante et profonde, surmontée d'une rosette terrestre de feuilles découpées et d'une tige ramifiée qui se termine par des ombelles florales. Le fruit est un diakène (Hmamouchi, 1999).

- $\quad$ Ecologie/Répartition : Rencontrée dans le Maroc oriental steppique, le Grand Alas, le Moyen Atlas y compris le Mont de Tazzeka, enclave à flore rifaine, les montagnes de Debdou à ghar Rouban, le Rif oriental et occidental, de la Moulouya à Oued Laou (Hmamouchi, 1999).

Ethnobotanique: Les parties de Carum carvi L. les plus utilisées en thérapeutique sont les feuilles et les fruits, sous forme de décoction et infusion. Ses huiles essentielles sont aussi utilisées mais à faible fréquence. La cuillerée est la dose recommandée et peut être prise pendant une journée, une semaine voire, un mois. Elle est sollicitée pour traiter les problèmes gastriques, et parfois pour des problèmes rhumatismaux (Daoudi et al., 2015-a ; El Hafian et al., 2014). Le fruit est utilisé comme carminatif, stomachique, antispasmodique, anti œdémateux, antidiurétique, apéritif, calmant, aphrodisiaque (Bellakhdar, 1997; Hmamouchi, 1999; Lahsissene et al., 2009). La décoction des semences est souvent recommandée en cas de douleurs abdominales et contre les vers intestinaux (Mouhib et El Omari, 1997); elle est aussi utilisée pour augmenter la sécrétion lactique (Sijelmassi, 1996), et pour aromatiser le pain (Chemat et al., 2004).

- Phytochimie: Les tests phytochimiques révèlent la richesse du carvi en tanins et plus précisément en tanins galliques. Les anthocyanes, représentant l'une des sous-classes des flavonoïdes, trouvé chez Carum carvi L. est l'un des puissants antioxydants nettoyant l'organisme des radicaux libres et maintenant une bonne circulation du sang. Nos résultats corroborent ceux de (Boukri, 2014).

La teneur la plus élevée en polyphénols totaux est celle du décocté, soit 64,86 \pm 0,69 mg EAG/g d'extrait; cette teneur est plus élevée en comparaison avec celle trouvée par ChristovaBagdassarian et al. (2013).

- $\quad$ Activité biologique : Le macérât du carvi à une concentration de $100 \mu \mathrm{g} / \mathrm{ml}$, est pourvu d'une activité antibactérienne vis-à-vis de Klebseilla pneumoniae (Daoudi et al., 2017- b). En effet, peu de travaux ont été menés sur les extraits du C. carvi L.; c'est plutôt son huile essentielle qui a été rapportée avoir une grande efficacité dans les traitements des pathologies infectieuses (Mohsenzadeh, 2007). 

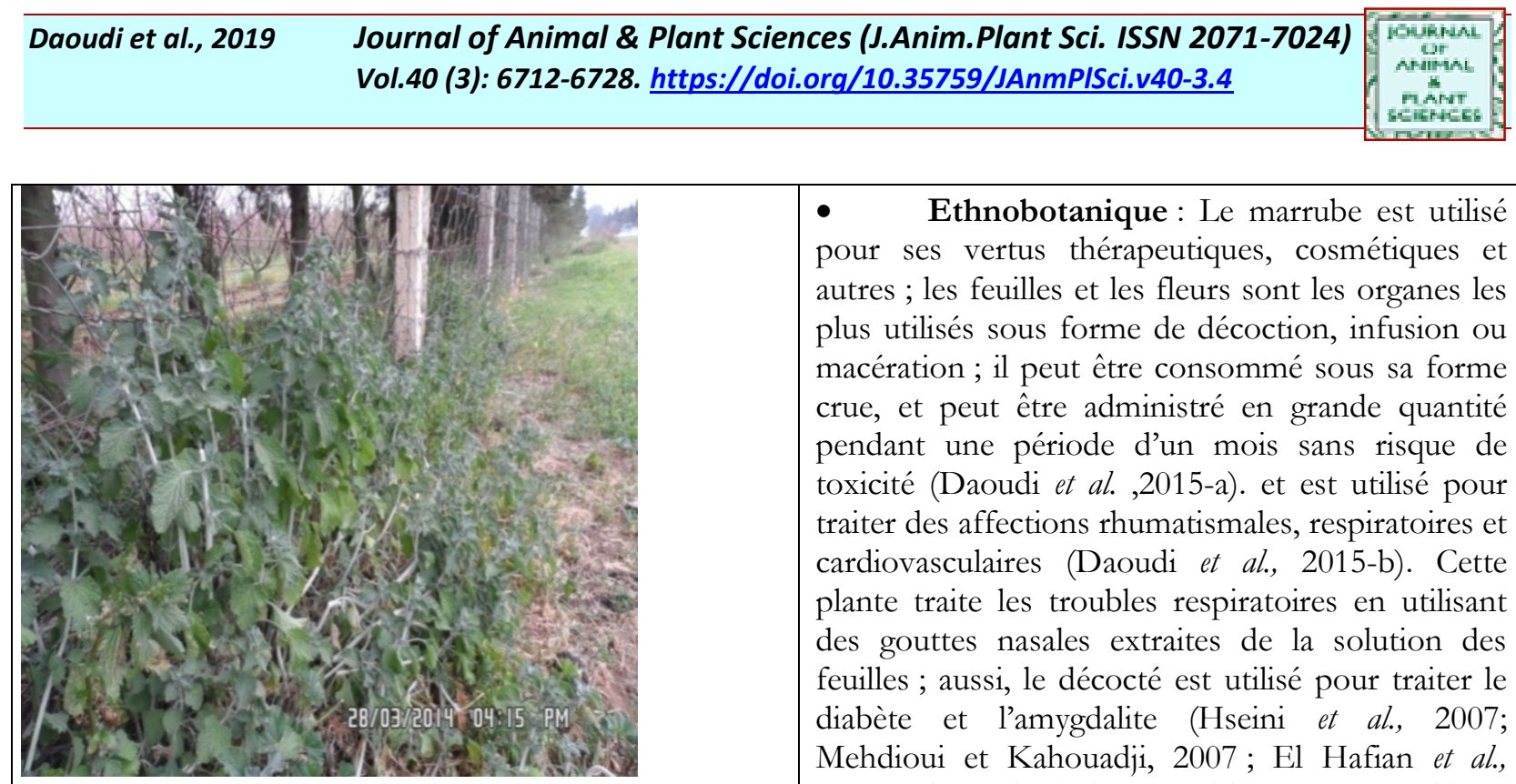

\section{Systématique}

- Embranchement : Spermaphytes

- Sous-embranchement : Angiospermes

- Classe : Dicotylédones

- Ordre : Lamiales

- Famille : Lamiacées

- Genre : Marrubium L.

Espèce : Marrubium vulgare $\mathrm{L}$.

- Nom français : Marrube blanc

- Nom arabe : مريوتة - mariouta

- Nom berbère : إفزئ-Ifzi

- Description botanique : Plante vivace de 30 à $60 \mathrm{~cm}$ de hauteur, à tiges quadrangulaires dressées, rameuses, couvertes d'un duvet blanchâtre. Les feuilles sont opposées, ovales ou arrondies, d'un vert cendré, blanchâtres au-dessous, gaufrées et inégalement crénelées. les fleurs, qui apparaissent en mai, sont petites, à l'aisselle de bractées ayant l'aspect de feuilles. Le fruit est un tétrakène (Bellakhdar, 2006; Fennane et al., 2007).

- Ecologie/Répartition : Le marrube blanc se rencontre dans les endroits incultes, les décombres, près des habitations et au bord des chemins; il se trouve sur les plaines, basses et moyennes montagnes. Bioclimats : Aride, semi-aride doux, semi-aride froid, subhumide et humide. Il est fréquent dans tout le Maroc (Bellakhdar, 2006; Fennane et al., 2007).

- $\quad$ Ethnobotanique : Le marrube est utilisé pour ses vertus thérapeutiques, cosmétiques et autres; les feuilles et les fleurs sont les organes les plus utilisés sous forme de décoction, infusion ou macération ; il peut être consommé sous sa forme crue, et peut être administré en grande quantité pendant une période d'un mois sans risque de toxicité (Daoudi et al. ,2015-a). et est utilisé pour traiter des affections rhumatismales, respiratoires et cardiovasculaires (Daoudi et al., 2015-b). Cette plante traite les troubles respiratoires en utilisant des gouttes nasales extraites de la solution des feuilles ; aussi, le décocté est utilisé pour traiter le diabète et l'amygdalite (Hseini et al., 2007; Mehdioui et Kahouadji, 2007 ; El Hafian et al., 2014). hypoglycémiante, sédative, stomachique (Hmamouchi, 1999), cette plante est aussi efficace en cas de fièvre typhoïde (Benkhnigue et al., 2010).

- Phytochimie : Le screening phytochimique de $M$. vulgare révèle la présence de tanins, de flavonoïdes, de terpénoides, de composés réducteurs et de dérivés anthracéniques; cela, concorde avec ceux de (Bouterfas et al., 2014). Cette plante contient un principe amer lactonique, la marrubiine, des alcools diterpiniques, un mucilage, des pectines, des sucres, une résine et des matières minérales (Bellakhdar, 2006).

- Activité biologique: L'huile essentielle de M. vulgare n'a aucun effet sur

Stapbylococus aureus, Escherichia coli et Klebseilla pneumoniae; les mêmes résultats ont été trouvés par (Ghedadba et al., 2015), et ceci malgré la présence de nombreux composants dans l'huile essentielle dont les principaux sont l' y-eudesmol, le $\beta$ citronellol, le citronellyle formate, le $\beta$-bisabolene, l' $\delta$-cadinene, l'isocaryophyllene et le germacrene. Certaines études ont confirmé l'activité antibactérienne puissante des HE de cette plante contre S. aureus (Bokaeian et al., 2014), cette différence peut être due à de nombreux facteurs, notamment, la période de cueillette qui peut affecter la composition chimique de la plante. 


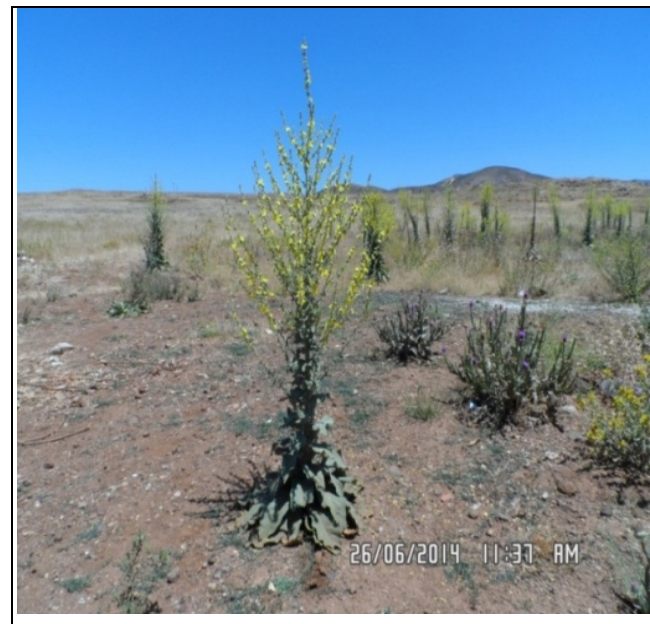

\section{Systématique}

- Embranchement : Spermaphytes

- Sous-embranchement : Angiospermes

- Classe : Dicotylédones

- Ordre : Scrophulariales

- Famille : Scrophulariacées

- Genre : Verbascum L.

- Espèce : Verbascum sinuatum L.

- Nom français : Molène sinuée

- Nom arabe : مصلح النضر - Maslahndar

- Nom berbère : مصلح :Maslah

Description botanique : Plante herbacée, bisannuelle hémicryptophyte (printemps et été). Feuilles alternes, oblongues ou ovales, à marges souvent entières crénelées ou sinuées, les basales en rosette, les caulinaires souvent sessiles et plus ou moins décurrentes. Fleurs solitaires ou en glomérules, axillaires, en grappe ou en panicule allongée. Fleurs subrésulières. Calice \pm profondément divisé en 5 segments (Bellakhdar 2006 ; Fennane et al., 2007).

- Ecologie/Répartition : L'espèce est fréquente dans les matorrals, les ermes, les steppes, les plaines et les basses et moyennes montagnes. Bioclimats : aride, semi-aride doux, semi-aride froid, subhumide et humide. Elle se rencontre dans toutes les divisions biogéographiques marocaines sauf le Maroc saharien (Fennane et al., 2007).
- Ethnobotanique : Verbascum sinuatum L. est utilisé pour ses vertus thérapeutiques; les feuilles, les racines et les fleurs sont les organes les plus utilisés. La décoction est le mode de préparation le plus courant, et la plante est administrée en petite quantité (une pincée) pour une période qui ne dépasse pas une semaine, vu le risque éventuel de toxicité (Daoudi et al., 2015-a). Cette plante est utilisée pour traiter des affections rhumatismales, dermatologiques et respiratoires (Daoudi et al., 2015-b). La racine est utilisée pour traiter les conjonctivites d'où son nom en arabe «Maslahndar» et aussi, de la cataracte; les feuilles sont utilisées pour usage externe, contre les boutons et comme anti-vermines (Bellakhdar, 1997). La plante entière est indiquée dans le traitement des rhumatismes et une macération des racines de la plante avec des graines de pois-chiche est conseillée en cas de fièvre typhoïde (Benkhnigue et al., 2010).

- $\quad$ Phytochimie : L'analyse phytochimique de cette plante révèle la présence de nombreux constituants chimiques, entre autres, des flavonoïdes, des stéroïdes, des alcaloïdes, des saponines, des glycosides monoterpènes, des mucilages. Ces résultats concordent avec ceux de (Tatli et Akdemir, 2004). L'huile essentielle de cette plante contient du limonène, du $\mathrm{D}$-fenchone, le 1,8cinéole, le $\beta$ caryophyllène, les $\alpha$-humulène, $\alpha$ pinène et de l'oxyde de piperitenone ; cette myriade de principes actifs peut être responsable d'une forte activité antioxydante et biologique (Karamian et Ghasemlou, 2013).

- $\quad$ Activité biologique : Les huiles essentielles de $V$. sinuatum L. utilisées contre Staphylococus aureus, Escherichia coli et Klebseilla pneumoniae, agents pathogènes responsables respectivement d'affections pulmonaires, gastriques et dermatologiques sont très actives; ceci est en accord avec les résultats rapportés par Sener et Dulger (2009). 


\begin{tabular}{lll}
\hline Daoudi et al., 2019 & $\begin{array}{l}\text { Journal of Animal \& Plant Sciences (J.Anim.Plant Sci. ISSN 2071-7024) } \\
\text { Vol.40 (3): 6712-6728. https://doi.org/10.35759/JAnmPISci.v40-3.4 }\end{array}$
\end{tabular}

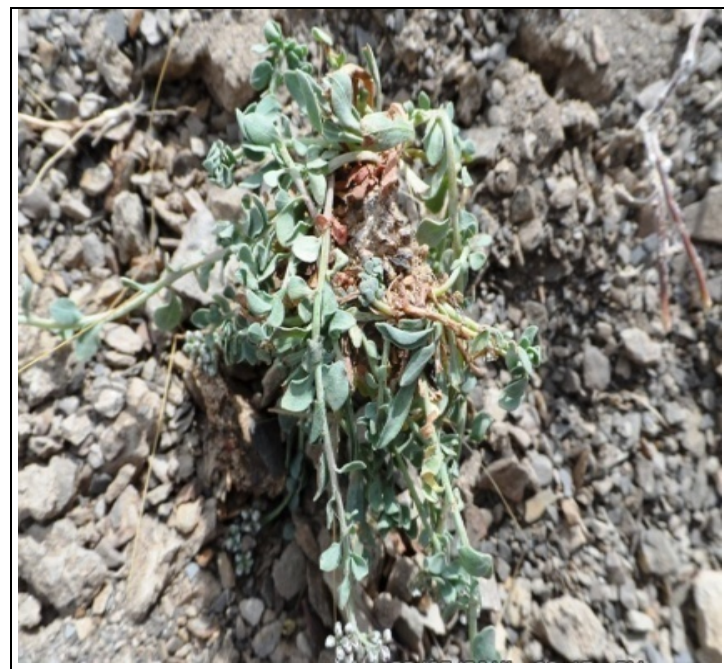

\section{Systématique}

- Embranchement : Spermaphytes

- Sous-embranchement : Angiospermes

- Classe : Dicotylédones

- Ordre: Caryphyllales

- Famille: Caryophylacées

- Genre: Corrigiola L.

-Espèce: Corrigiola telephiifolia Pourret.

- Nom français : Corrigiole

- Nom arabe : سر غينة:Serguina

- Nom berbère -Tassarghint

\section{$22 / 05 / 2014$}

- Description botanique : Plante herbacée. Les feuilles caulinaires oblongues-lancéolés, spatulées ou ovales, sont un peu épaisses, les racines sont odorantes. Inflorescence en glomérules axillaires et terminaux. Fleur 5-mère. Périanthe blanc ou un peu rosé, sensiblement aussi long que les sépales. 5 étamines plus courtes que les pétales. Anthères blanchâtres. 3 stigmates. Akènes: trigones, crustacés, inclus dans le périanthe persistant. Hémi-cryptophyte (printemps et été). (Bellakhdar 2006 ; Fennane et al., 2007).

- Ecologie/Répartition : La corrigiole se trouve sur les terrains pierreux et sablonneux des plaines et des montagnes. Bioclimats: semi-aride doux, semi-aride froid, subhumide. Elle est rencontrée dans l'Anti Atlas (Siroua), Haut Atlas, Moyen Atlas, Maroc atlantique moyen, Maroc atlantique nord et le Rif (Fennane et al., 1999).
- Ethnobotanique : La corrigiole est utilisée surtout en cosmétique, mais certaines personnes l'utilisent pour ses vertus thérapeutiques ; la racine est l'organe le plus utilisé et la décoction est le mode de préparation le plus courant. La racine réduite en poudre est administrée en cuillerée pour une période allant d'une journée jusqu'à la guérison (Daoudi et al., 2015-a). Cette plante est utilisée pour traiter des pathologies respiratoires, gastriques et dermatologiques, urogénitales et des affections neurologiques, microbiennes ORL et typhoïdes (Daoudi et al., 2015-b). Elle est utilisée aussi contre les allergies (Lahsissene et al., 2009 ; El Hafian et al., 2014).

En outre, les racines de serghina sont très utilisées dans les fumigations et ont la réputation de tenir à distance les mauvais génies (Bellakhdar, 1997).

- Phytochimie : Le screening phytochimique de la poudre de C. telephifolia Pourret révèle la présence d'alcaloïdes, de quantités relativement moyennes en tanins et en flavones, une richesse en stérols et triterpènes et la présence remarquable de saponines; pour cette dernière classe, il est rapporté que les espèces appartenant à la famille des Caryophyllaceae sont souvent riches en saponines et principalement les saponines triterpéniques (Timite, 2012). Le dosage des polyphénols montre que les concentrations les plus élevées sont observées dans le macéré de $C$. telephifolia Pourret. avec 58,5 $\pm 0,76 \mathrm{mg}$ équivalent d'acide gallique/g d'extrait.

- Activité biologique : le macérât de la corrigiole est légèrement actif sur Escherichia coli (Daoudi et al., 2017-b) ; ceci est en accord avec les travaux de Doudach et al. (2013).Ce pouvoir inhibiteur pourrait être attribué à l'abondance de saponines. 


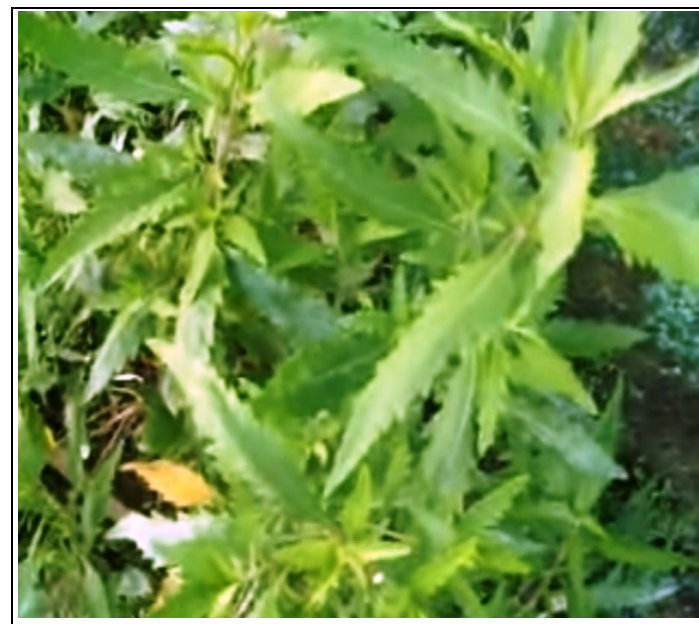

- Ethnobotanique: Le chénopode est utilisé surtout en thérapeutique ; les feuilles et les fruits sont les organes les plus utilisés, la macération est le mode de préparation le plus courant, les feuilles séchées ou réduites en poudre sont administrées en quantités importantes pour une journée, mais, la plante peut être utilisée pour une plus longue période jusqu'à la guérison. Toutefois, pour des raisons de sécurité, il faut l'administrer avec des précautions. Cette plante est utilisée pour traiter des pathologies rhumatismales, respiratoires et gastriques (Daoudi et al., 2015-a) et utilisée aussi pour traiter des maladies de l'appareil circulatoire (Hseini et al., 2007), le traitement d'affections gastro-intestinales, la typhoïde et la migraine (Bellakhdar, 1997; Lahsissene et al., 2009). Les graines de cette plante sont utilisées comme vermifuge, contre les abcès buccaux, les ulcérations et les plaies purulentes, par application locale (Bellakhdar, 1997) et l'huile essentielle a montré une importante activité antalgique de type non morphinique (Ongoka et al., 2003).

- Nom français : Chénopode - Nom arabe : مخينزة

Mkhinza - Nom berbère: تمكوتTamagout

\section{$10 / 03 / 2013$}

- Description botanique: Plante annuelle ou vivace, herbacée ou suffrutescente, glabre, farineuse ou poilue-glanduleuse. Tiges plus ou moins sillonnées. Feuilles à dents espacées sur les marges, nervures peu saillantes en dessous. Inflorescence en épi ou en panicule de glomérules axillaires. Fruit : akène à péricarpe scarieux. Graine horizontale ou verticale, lenticulaire, lisse ou ponctuée noirâtre et luisante (Fennane et al., 1999).

- Ecologie/Répartition: Espèce naturalisée ; abondante dans les milieux incultes frais, fossés, haies et alluvions sablonneux. Bioclimats : aride semi-aride doux, subhumide. Elle se trouve dans le Maroc saharien, Haut Atlas, Maroc atlantique moyen, Maroc atlantique nord, plateaux du Maroc oriental, le littoral de la méditerranée et le Rif (Fennane et al., 1999)
- Phytochimie : Le screening phytochimique de la poudre de C. ambrosioides L. montre la présence d'alcaloïdes et de quantités relativement moyennes en tanins et stérols et triterpènes). Le dosage des polyphénols des différents extraits révèle que, comparé à l'infusé et au macérât, le décocté de $C$. ambrosioides $\mathrm{L}$. présente la quantité équivalente d'acide gallique en mg par $1 \mathrm{~g}$ d'extrait la plus importante, soit : 82,42 Qteq Ag mg / 1g d'extrait (Daoudi et al., 2017-a)

- $\quad$ Activité biologique : Cette plante est dotée d'une activité antifongique attestée par l'inhibition de la croissance mycélienne des pathogènes: Aspergillus brasiliensis, Fusarium oxysporum, Penicillium expansum et Rhizopus stolonifer (Daoudi et al., 2017- a). 


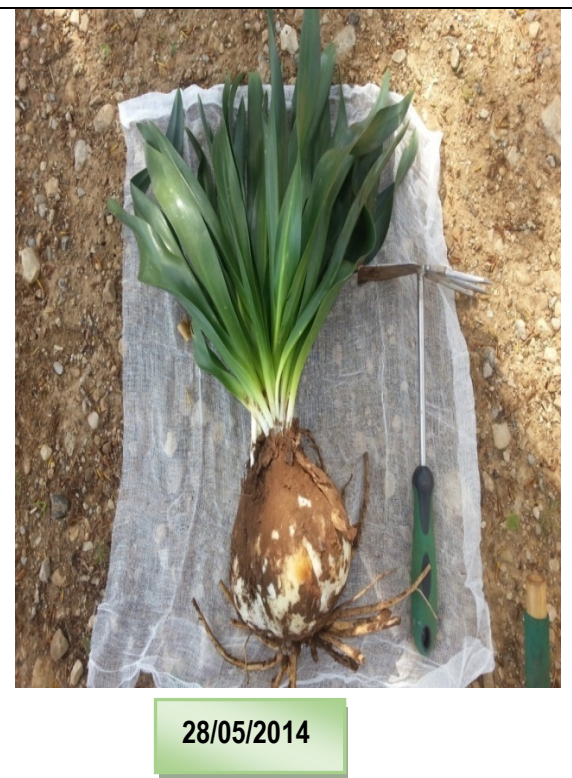

- Description botanique: Plante géophyte, herbacée, vivace. La tige robuste peut atteindre $1.50 \mathrm{~m}$. Les feuilles entières, lancéolées, longues de 50 à $80 \mathrm{~cm}$. Elle apparaît au printemps en touffes, les fleurs en grappes, serrées, sont accompagnées de petites bractées dialyphylles. Le fruit est une petite capsule à trois loges avec des graines aplaties. La partie souterraine est constituée par un bulbe formé d'écailles emboitées, insérées sur un plateau qui porte de nombreuses racines, sur sa face inférieure (Hmammouchi, 1999).

- Ecologie/Répartition: L'Urginée maritime croît dans les steppes, les broussailles et les pâturages pierreux ou sablonneux des plaines et des basses montagnes. Spontanée dans la région méditerranéenne, elle existe en abondance sur le territoire beniurgin (d'où le nom urginea). Au Maroc, elle existe au niveau du Rif (Beni znassen), en montagne jusqu'à $1400 \mathrm{~m}$, au niveau du Moyen Atlas, la région de Fès et de Khmisset et la plaine atlantique. (Fennane et al., 2014). lique : Urginea maritima (L.) tout en thérapeutique ; le fruit utilisé et la décoction est le in le plus courant; aussi, le $t$ en poudre est administré en pour une journée seulement, sécurité puisque cette plante oxicité.

indiquée pour traiter des maux (Daoudi et al., 2015-a), ss vertus, et on l'utilise sous pour traiter les douleurs de broyage et filtration du bulbe 4). Aussi, elle est utilisée pour yies digestives (Mehdioui et les problèmes cardiaques, la mouchi, 1999), et est 1 commandée pour traiter l'hépatite (Bellakhdar, 1997 ; Benkhnigue et al., 2010).

- Phytochimie : Le screening phytochimique de la poudre d'U. maritima révèle la présence d'hétérosides, de mucilages et de lipides à des quantités relativement importantes; aussi on note la présence de tanins catechiques et certains dérivés phénoliques. Le dosage des polyphénols des différents extraits a révélé que le macérât d'U. maritima recèle une quantité élevée en polyphenols (60,90 Qteq Ag mg / 1g d'extrait). (Daoudi et al., 2017- a).

- Activité biologique: L'extrait aqueux d'U. maritima indique une activité antifongique positive sur Aspergillus brasiliensis et Fusarium oxysporum, (Daoudi et al., 2017-a ); ceci est lié la présence d'une glycoprotéine localisée dans la paroi cellulaire de l'ampoule, qui inhibe complètement la germination des spores et la croissance des hyphes de (Deepak et al., 2003).

\section{REFERENCES BIBLIOGRAPHIQUES}

Benbrahim KF, Ismaili M, Benbrahim SF and Tribak A : 2004.Problèmes de dégradation de l'environnement par la désertification et la déforestation: impact du phénomène au Maroc. Science et changements planétaires/Sécheresse, 15(4), 307-320.
Boujrouf S : 2004, Tourisme de montagne au Maroc: enjeux de la durabilité. Le tourisme durable, réalités et perspectives marocaines et internationales. Marrakech, 273-284.

Bokaeian M, Saboori E, Saeidi S, Niazi A, Amini-Borojeni N, Khaje H. and Bazi S:2014. Phytochemical Analysis, 
Antibacterial Activity of Marrubium vulgare $L$ against Staphylococcus aureus in vitro. Zahedan Journal of Research in Medical Sciences 16(10):60-64.

Belkassam A, Zellagui A, Gherraf N, Lahouel $M$.and Rhouati S:2011. Essential oil composition of Algerian Ruta Montana (Clus.) L. and its antibacterial effects on microorganisms responsible for respiratory infections. Advances in Natural and Applied Sciences 5(3):264269.

Bellakhdar J : 1997. La pharmacopée marocaine traditionnelle. Médecine arabe ancienne et savoirs populaires. Editions Le Fennec, Casablanca. 764 pp: Ibis Press.

Bellakhdar J, Claisse R, Fleurentin J. and Younous C: 1991. Repertory of standard herbal drugs in the Moroccan pharmacopoea. Journal of ethnopharmacology 35(2):123-143.

Bellakhdar J : 2006. Plantes médicinales au Maghreb et soins de base: précis de phytothérapie moderne: Casablanca, Editions Le Fennec. 385 p.

Benabderrahmane M, Benali M, Aouissat $\mathrm{H}$. and Bueso MJJ: 2009. Activite antimicrobienne des huiles essentielles de Pistacia atlantica Desf. de l'Algerie. Phytothérapie 7(6):304-308.

Benhassaini H, Mehdadi Z, Hamel L.and Belkhodja M : 2007. Phytoécologie de Pistacia atlantica Desf. subsp. atlantica dans le Nord-Ouest algérien. Science et changements planétaires/Sécheresse, 18(3), 199-205.

Benkhnigue O, Zidane L, Fadli M, Elyacoubi H, Rochdi A. and Douira A:2010. Etude ethnobotanique des plantes médicinales dans la région de Mechraâ Bel Ksiri (Région du Gharb du Maroc).Acta Botanica Barcinonensia 53:191-216.

Boukri NEH : 2014. Contribution à l'étude phytochimique des extraits bruts des épices contenus dans le mélange Ras-elhanout. Thèse doctorat. Université Kasdi Merbah. Ouargla 99 p.
Bouterfas K, Mehdadi Z, Latreche A. and Aouad L : 2014. Pouvoir antimicrobien des flavonoïdes extraits des feuilles de Marrubium vulgare $L$. en provenance du mont de Tessala (Algérie occidentale). Phytothérapie 12(1):6-14.

Chemat S, Lagha A, AitAmar H, Bartels PV. and Chemat F: 2004. Comparison of conventional and ultrasound-assisted extraction of carvone and limonene from caraway seeds. Flavour and Fragrance Journal 19(3):188-195.

Christova-Bagdassarian VL, Bagdassarian KS. and Atanassova M:2013. Phenolic profile, antioxidant and antimicrobial activities from the Apiaceae family (dry seeds). Mintage Journal of Pharmaceutical \& Medical Sciences 2(4):26-31.

Daoudi A, Boutou H, Zair T, Ibijbijen J. and Nassiri L : 2013. Etude ethnobotanique du pistachier de l'Atlas, Pistacia atlantica, dans la ville de Meknès-Maroc. Science Lib

Daoudi A, Nassiri L, Ibijbijen J. and Boukil A : 2014. Etude ethnobotanique du Pyrèthre d'Afrique " Anacyclus pyrethrum L." dans le cercle Meknès, El Hajeb, Khénifra, Azrou et Ifrane Maroc-. Science Lib 6:26.

Daoudi A, Bachiri L, Bammou M, Ibijbijen J, Nassiri L, 2015.a. Etude ethnobotanique au Moyen Atals Central. European Scientific Journal 11(24).

DaoudiA, Bammou M, Zarkani S, Slimani I, Ibijbijen J. and Nassiri L: 2015 .b. Étude ethnobotanique de la flore médicinale dans la commune rurale d'aguelmouss province de khénifra (Maroc). Phytothérapie 13(2):1-9.

Daoudi A, Sabiri M, Bammou M, Zair T, Ibijbijen J. and Nassiri L: 2015, c. Valorisation des extraits de trois espèces du genre Urtica: Urtica urens L., Urtica membranacea Poiret et Urtica pilulifera L. Journal of Applied Biosciences 87(1):8094-8104. 
Daoudi A, Hrouk H, Belaidi R, Slimani I, Ibijbijen J. and Nassiri L:2016. Valorisation de Ruta montana et Ruta chalepensis: Etude ethnobotanique, Screening phytochimique et pouvoir antibactérien. . Journal of Materials and Environmental Science 7:10.

Daoudi A, Bammou M, Haloui Z, Ibijbijen J. and Nassiri L: 2017. a. Activite Antifongique Des Extraits Aqueux De Calendula Officinalis L, Urginea Maritima (L.) Baker et Chenopodium Ambrosioides L. European Scientific Journal, ESJ, 13(24), 483.

Daoudi A, Bammou M, Ibijbijen J. and Nassiri L: 2017.b. Antibacterial Activity of Aqueous Extracts of Anacyclus Pyrethrum (L) Link and Corrigiola Telephiifolia Pourr. From the Middle Atlas Region-Morocco. European Scientific Journal, ESJ, 13(33), 116.

Deepak A, Thippeswamy G, Shivakameshwari M. and Salimath BP: 2003.Isolation and characterization of a $29-\mathrm{kDa}$ glycoprotein with antifungal activity from bulbs of Urginea indica. Biochemical and Biophysical research communications 311(3):735-742

El Hafian M, Benlandini N, Elyacoubi $H$, Zidane L, Rochdi A:2014. Étude floristique et ethnobotanique des plantes médicinales utilisées au niveau de la préfecture d'Agadir-Ida-Outanane (Maroc). Journal of Applied Biosciences 81(1):7198-7213.

El Azzouzi H, Soro A, Elhilali F, Bentayeb A, El Belghiti MA. and Zair T:2014. Phytochemical study of Anacyclus pyrethrum (L.) of Middle Atlas (Morocco), and in vitro study of antibacterial activity of pyrethrum. Advances in Natural and Applied Sciences 8(8):131-141.

Farag MA, Weigend M, Luebert F, Brokamp G.and Wessjohann LA: 2013. Phytochemical, phylogenetic, and antiinflammatory evaluation of 43 Urtica accessions (stinging nettle) based on
UPLC-Q-TOF-MS metabolomic profiles. Phytochemistry 96:170-183.

Fennane M, Ibn-Tattou M, Ouyahya A, Mathez J .and El Oualidi J : 1999. Flore pratique du Maroc. Vol. 1. Pteridophyta, Gymnospermae, Angiospermae (Lauraceae-Neuradaceae): Inst. Scientifique. 558p.

Fennane M, Ibn-Tattou M, Ouyahya A .and El Oualidi J : 2007. Flore pratique du Maroc. Vol. 2. Angiospermae (Leguminosae-Lentibulariaceae): Inst. Scientifique. 636p.

Fennane M, Ibn-Tattou M and El oualidi J : 2014. Flore pratique du Maroc. Vol. 3. Dicotyledones, Monocotyledones: Inst. Scientifique. $755 \mathrm{p}$.

Ghédira K and Goetz P: 2016. Calendula officinalis L. (Asteraceae): souci. Phytothérapie 14(1):62-67.

Giner-Larza EM, Máñez S, Recio MC, Giner RM, Prieto JM, Cerdá-Nicolás M.and Ríos JL, :2001. Oleanonic acid, a 3oxotriterpene from Pistacia, inhibits leukotriene synthesis and has antiinflammatory activity. European journal of pharmacology 428(1):137-143.

Ghalem BR and Benali M, 2009. Bactericidal activity of Pistacia atlantica. Desf mastic gum against certain pathogens. African Journal of Plant Science 3(1):013-015.

Ghedadba N, Hambaba L, Ayachi A, Aberkane M, Bousselsela H. and OueldMokhtar S: 2015. Polyphénols totaux, activités antioxydante et antimicrobienne des extraits des feuilles de Marrubium deserti de Noé. Phytothérapie 13(2):118-129.

Hamimed S : 2009. Caractérisation chimique des principes à effet antidermatophyte des racines d'Anacyclus pyrethrum L. Mémoire de troisème cycle. Université de Mentouri Constantine. 166 p.

Hseini S, Kahouadji A, Lahssissene H.and Tijane M : 2007. Analyses floristique et ethnobotanique des plantes vasculaires médicinales utilisées dans la région de 
Rabat (Maroc occidental). Lazaroa 28:93-100.

Hmamouchi M : 1999. Les plantes médicinales et aromatiques marocaines. Rabat.: Editions Fedala.389p.

Karamian R and Ghasemlou F. 2013. Total phenolic content, antioxidant and antibacterial activities of three Verbascum species from Iran. Journal of Medicinal Plants and By-Products 2(1):43-51.

Lahsissene H, Kahouadji A.and Hseini S: 2009. Catalogue des plantes medicinales utilisées dans la region de Zaër (Maroc Occidental). Lejeunia, Revue de Botanique (0457-4184):30.

Maamri S : 2008. Etude de pistacia atlantica de deux régions de sud Algérien: dosage des lipides, dosage des polyphénols, essais antileishmaniens. Thèse doctorat. Université de Boumerdès, Algerie 98 p.

Mouhib M and El Omari Z : 1997. Nos plantes médicinales. Emplois en médecine moderne, en homéopathie et en médecine populaire. Edition. Fès, Maroc. 221 p.

Mehdioui R and Kahouadji A : 2007. Etude ethnobotanique auprès de la population riveraine de la forêt d'Amsittène: cas de la Commune d'Imi n'Tlit (Province d'Essaouira). Bulletin de l'Institut scientifique, Rabat, section Sciences de la vie 29:11-20.
Mohsenzadeh M: 2007. Evaluation of antibacterial activity of selected Iranian essential oils against Staphylococcus aureus and Escherichia coli in nutrient broth medium. Pak J Biol Sci 10(20):3693-3697.

Sijelmassi A : 1996. Les plantes médicinales du Maroc. Casablanca, Morocco: Editions Le Fennec 285p.

Selles C, Dib MEA, Allali H.and Tabti B: 2012. Evaluation of antimicrobial and antioxidant activities of solvent extracts of Anacyclus pyrethrum L., from Algeria. Mediterranean Journal of Chemistry 2(2):408-415.

Sener A and Dulger B: 2009. Antimicrobial activity of the leaves of Verbascum sinuatum L. on microorganisms isolated from urinary tract infection. African Journal of Microbiology Research 3(11):778-781.

Tatli I and Akdemir Z: 2004. Chemical constituents of Verbascum L. species. FABAD J Pharm Scieces 29:93-107.

Timite G : 2012. Isolement et caractérisation des saponosides de plantes de la famille des Alliaceae, Caryophyllaceae et Polygalaceae, et évaluation de leurs activités cytotoxiques sur cellules tumorales. Thèse de doctorat. Université de Bourgogne.32 
Annexe 1: Questionnaire d'enquête ethnobotanique

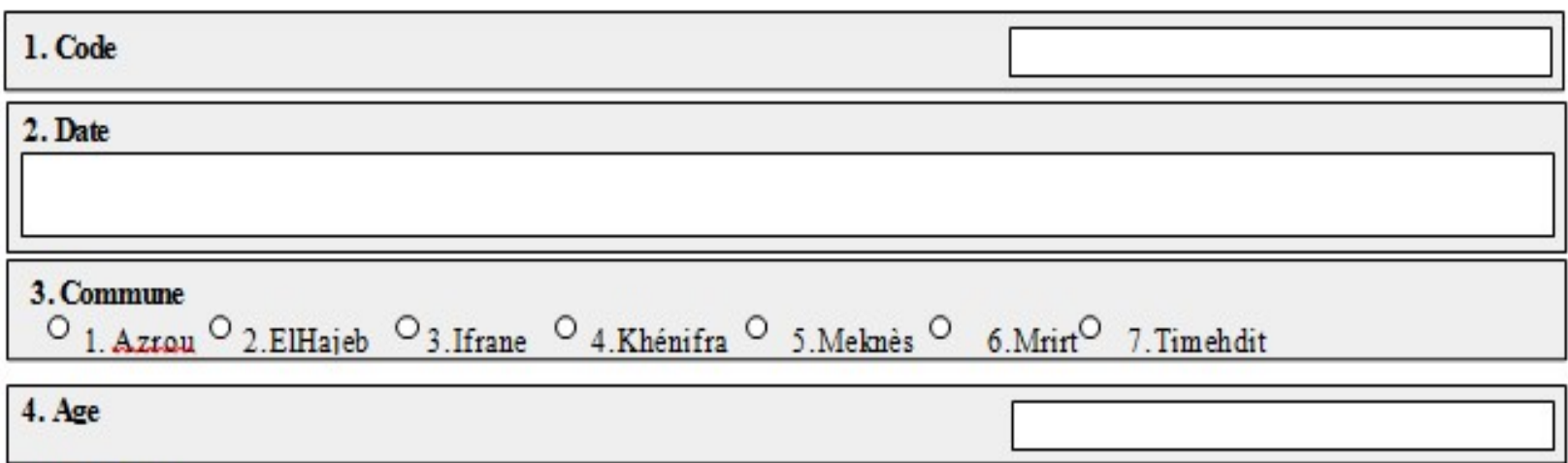

\section{Profession}

O 1. Droguiste O2.Herboriste $\mathrm{O}$ 3.Tradipraticien

\section{Sexe \\ O 1. Féminin $O$ 2.Masculin}

\begin{tabular}{|c|c|c|c|}
\hline $\begin{array}{l}\text { 7. Nireau } \\
0 \text { 1. Néant }\end{array}$ & 2.Primaire & O3.Secondaire & O 4.Universitaire \\
\hline 8. Localité & & & \\
\hline O 1. Nomade & O 2.Vile & O 3.Douar & 4.VAllage \\
\hline 9. Famille & & & \\
\hline
\end{tabular}

\section{Genre}

\begin{tabular}{|l|}
\hline 1 . Es pèce \\
\hline
\end{tabular}

\begin{tabular}{|l|}
\hline 12. Arabe \\
\hline \\
\hline 13. Francais \\
\hline \\
\hline 14. Tamazigh \\
\hline
\end{tabular}

\section{Type}

$\square$ 1. Culivié $\square 2$ Sauvage

Vous pones cocher plusieurs 


\section{Usage \\ $\square$ 1. Cosmétique $\quad \square \quad$ 2.Thérapertique $\square \quad$ 3.Autre \\ Vous pouvez cocher plusieurs cases.}

\section{Etat}

$\square$ 1. Frais $\square \quad$ 2.Séché

Vous pouvez cocher plusieurs cases.

\section{Partie utilisée}

$\square$ 1. Feuilles $\square$ 2.Fruits $\square$ 3.Racine $\square$ 4.Tige $\square$ 5.Grames $\square$ 6.Résine $\square$ 7.Fleurs $\square$ 8.Autres $\square$ 9. Capsules

Vous powvez cocher plusieurs cases (8 au maximum)

\section{Forme d'emploi}

$\square$ 1. Poudre $\square$ 2.Tisane $\square$ 3.Huileessentielle $\quad \square$ 4.Extrait

Vous pouvez cocher plusieurs cases.

\section{Mode de préparation}

$\square$ 1. Décoction $\quad \square$ 2.Infusion $\quad \square$ 3.Macération $\quad \square \quad \square$ 4.Cru $\quad \square$ 5.Cuit $\quad$ 6.Autre

Vous powvez cocher plusieurs cases.

\section{Dose utilisée}

$\square$ 1. Cuillerée $\square$ 2.Pincée $\square$ 3.Poignée

Vous pouvez cocher plusieurs cases.

\section{Mode d'administration}

$\square$ 1. Oral $\square$ 2.Massage $\square$ 3. Rinçage $\square$ 4.Badigeonnage $\square$ 5.Autre

Vous pouver cocher plusieurs cases.

\section{Durée d'utilisation}

$\square$ 1. Un jour $\quad \square \quad$ 2.Unesemaine $\square$ 3.Un mois $\square$ 4.Jusqu'à guérison

Vous pouver cocher plusieurs cases.

\section{Maladie traitée}

\begin{tabular}{|ll|ll|l|}
\hline$\square$ Affections dermatologiques & $\square$ & Affections respiratoires & $\square$ & Affections cardio-vasculaires \\
\hline$\square$ Affections uro-gennitales & $\square$ & Affections osté-articulaires & $\square$ & Affections du tube digestif \\
\hline$\square$ Affections des glandes & $\square$ & Affections neurologiques & $\square$ & Affections métaboliques \\
\hline$\square$ Annexes du tube digestif & $\square$ & Affections ophtalmiques & $\square$ & Affections bucco-dentaires \\
\hline
\end{tabular}

Vous powver cocher plusieurs cases (10 aumaximum)!

\section{Toxicité de la plante}

$\mathrm{O}_{1 . \text { Oui }} \mathrm{O}_{2 . \mathrm{Non}}$ 